\title{
Atypical magnetic resonance imaging appearance of biopsy-proven spinal metastases in a trauma patient
}

Singapore Med J 2019; 60(7): 381-382 https://doi.org/10.11622/smedj.2019077

Dear Sir,

We report a case of a 75-year-old man who presented with back pain after a fall. Radiography revealed an L1 vertebral compression fracture, which was confirmed on subsequent magnetic resonance (MR) imaging of the spine. Numerous unsuspected, wellcircumscribed focal lesions, which were uniformly hypointense on both T1- and T2-weighted MR imaging sequences relative to the marrow (Fig. 1), were noted in the spine. While most of the lesions were occult, a few were appreciated as subtle hyperintense foci on short tau inversion recovery (STIR) sequence. The features were suggestive of predominantly sclerotic lesions, such as sclerotic metastases. However, computed tomography (CT) of the abdomen and pelvis, performed concurrently for evaluation of sepsis, revealed a pristine spine without sclerotic lesions or any features to suggest bony metastases (Fig. 1). We noted a cirrhotic liver with a focal hepatic mass on CT, which was suspicious for hepatocellular carcinoma (subsequently definitively characterised on dedicated MR imaging of the liver). Hence, a radiological diagnosis of possible skeletal metastases from a hepatocellular carcinoma was made. However, there was clinical scepticism, as CT did not reveal any corresponding sclerotic bony lesions.

Review of the limited images of the spine on MR imaging of the liver (subsequently performed with contrast) also revealed foci of restricted diffusion, as well as post-contrast enhancement (Fig. 2) corresponding to the focal lesions seen on the initial unenhanced MR images of the spine; this further favoured the diagnosis of metastatic lesions. Subsequent bone marrow biopsy confirmed the diagnosis of metastatic hepatocellular carcinoma.

The bone is the third most common site of metastasis after the lung and liver; the spine is the most commonly involved site in the skeletal system, ${ }^{(1)}$ and its diagnosis has significant implications in terms of staging, therapeutic decision-making and prognostication. (2) Imaging features of skeletal metastasis have been well characterised and typically appear as lytic, sclerotic or mixed lesions.
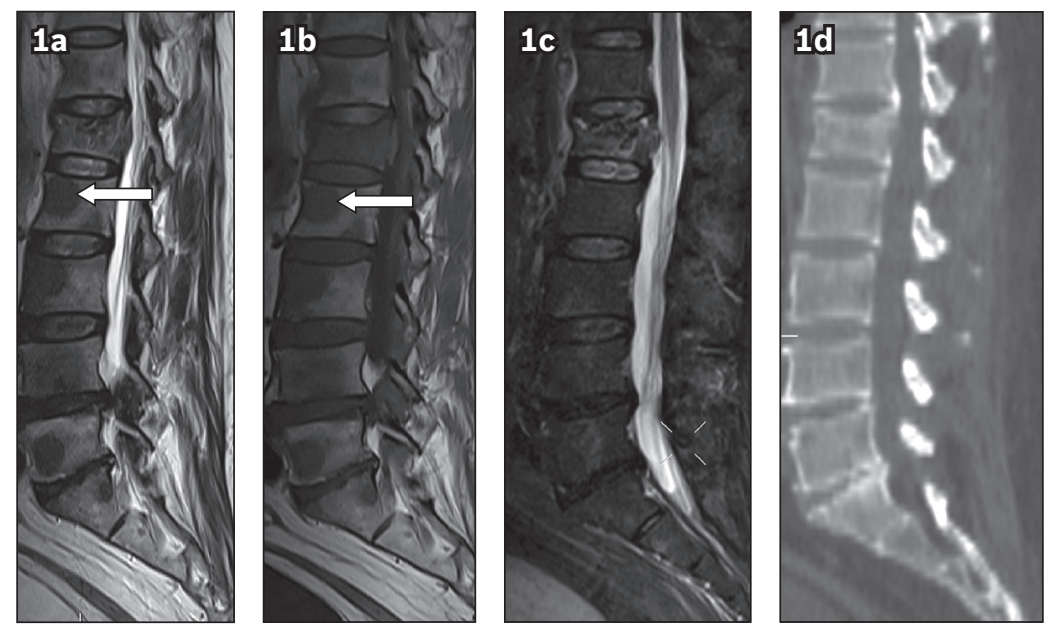

Fig. 1 Sagittal (a) T1-W, (b) T2-W and (c) short tau inversion recovery (STIR) images of the lumbar spine show compression fracture of the L1 vertebral body with multiple T1-W and T2-W hypointense focal lesions, most of which are occult on STIR image. One such lesion is noted in the L3 vertebral body (arrow in a \& b). (d) Sagittal CT image of the lumbar spine shows an L1 vertebral fracture and no corresponding sclerotic focal lesions.
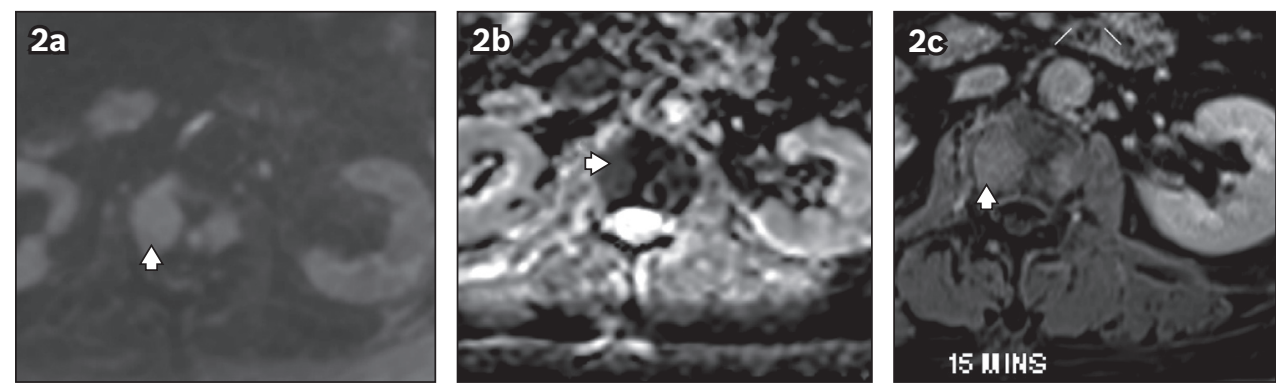

Fig. 2 (a) Axial diffusion-weighted, (b) apparent diffusion coefficient and (c) post-contrast (T1 fat-saturated) MR images of the abdomen at the L3 vertebral level show the corresponding focal lesion (arrowhead) as focus of restricted diffusion and homogeneous enhancement. 
Characteristically, on MR imaging, lytic metastases appear as T1 hypointense and T2 hyperintense focal lesions, with signal prolongation in STIR sequences. In particular, hepatic metastases demonstrate a predilection toward soft tissue formation. ${ }^{(3)}$ Sclerotic metastases classically show hypointense signal on T1-weighted, T2-weighted and STIR images. Post-contrast imaging with gadolinium complements these images by better demonstrating subtle lesions and delineating the associated soft tissue component in cases of spinal metastasis. ${ }^{(4)}$ In contrast to common cases where T1- and T2-weighted hypointensities are ascribed to sclerosis, in rare instances such as the present case, the reduced signal may also be indicative of tumoral hypercellularity with increased nuclear cytoplasmic ratios as noted on histopathology. This is a key factor that helps radiologists recognise apparently 'sclerotic' lesions seen on MR imaging without corresponding sclerotic lesions on CT, and prompts them to not dismiss these as nonspecific signal abnormalities. Active pursuance of these lesions for contrast-enhancement characteristics and associated soft tissue, if present, is highly recommended, as they might represent metastatic high-grade/hypercellular tumour foci. Hypercellular tumoral foci are also likely to demonstrate restricted diffusion; thus diffusion-weighted imaging may need to be added to the standard imaging protocol in addition to post-contrast sequences.

In conclusion, MR imaging is highly sensitive and known to detect metastasis as marrow signal abnormality well before the appearance of similar lytic or sclerotic changes on CT. The learning point in our case is that high-grade soft tissue/hypercellular spinal metastases may occasionally depict low T2-weighted signal as opposed to the typical T2-weighted hyperintense signal, and this is likely due to high nuclear-cytoplasmic ratios. Therefore, such low T2-weighted signal lesions must be further evaluated with diffusion-weighted and post-contrast imaging, along with histopathological correlation, before they are dismissed as nonspecific marrow signal abnormalities, especially in the absence of concomitant sclerotic lesions on CT.

Yours sincerely,

Pallavi Byrapu' ${ }^{1}$, Sindhu $\underline{\text { John' }}^{1}$

${ }^{1}$ Department of Radiology, Changi General Hospital, Singapore. drpallavichowdary@gmail.com

\section{References}

1. Macedo F, Ladeira K, Pinho F, et al. Bone metastases: an overview. Oncol Rev 2017; 11:321.

2. Kintzelé L, Weber MA. [Imaging diagnostics in bone metastases]. Radiologe 2017; 57:113-28. German.

3. Kim S, Chun M, Wang H, et al. Bone metastasis from primary hepatocellular carcinoma: characteristics of soft tissue formation. Cancer Res Treat 2007; 39:104-8.

4. Vanel D. MRI of bone metastases: the choice of the sequence. Cancer Imaging 2004; 4:30-5. 